Rabaska

Revue d'ethnologie de l'Amérique française

\title{
Alain (Allan) Kelly (1903-2008)
}

\section{Ronald Labelle}

Volume 7, 2009

URI : https://id.erudit.org/iderudit/038347ar

DOI : https://doi.org/10.7202/038347ar

Aller au sommaire du numéro

Éditeur(s)

Société québécoise d'ethnologie

ISSN

1703-7433 (imprimé)

1916-7350 (numérique)

Découvrir la revue

Citer ce document

Labelle, R. (2009). Alain (Allan) Kelly (1903-2008). Rabaska, 7, 141-144.

https://doi.org/10.7202/038347ar

Ce document est protégé par la loi sur le droit d'auteur. L'utilisation des services d'Érudit (y compris la reproduction) est assujettie à sa politique d'utilisation que vous pouvez consulter en ligne.

https://apropos.erudit.org/fr/usagers/politique-dutilisation/
Cet article est diffusé et préservé par Érudit.

Érudit est un consortium interuniversitaire sans but lucratif composé de l'Université de Montréal, l'Université Laval et l'Université du Québec à Montréal. Il a pour mission la promotion et la valorisation de la recherche. https://www.erudit.org/fr/ 


\section{Alain (Allan) Kelly (1903-2008)}

Le $1^{\text {er }}$ octobre 2008, l'Acadie a perdu un des derniers héritiers d'une tradition orale remontant à des siècles. Le premier numéro de la revue Rabaska (2003) comprenait un article consacré à Alain Kelly ( « Choisir son identité culturelle : le cas d'Allan/Alain Kelly »). J'y expliquais comment ce chanteur traditionnel avait opté de s'identifier aux Acadiens de son milieu, alors qu'avec un nom irlandais et une culture bilingue, il aurait pu facilement s'intégrer à la majorité anglophone du NouveauBrunswick.

Le choix qu'avait fait Kelly a eu des répercussions importantes en ethnologie acadienne, parce qu'entre 1958 et 2002 il a été l'informateur par excellence, transmettant non seulement un bagage extrêmement impressionnant de chansons traditionnelles (environ 300 titres différents), mais aussi des connais-

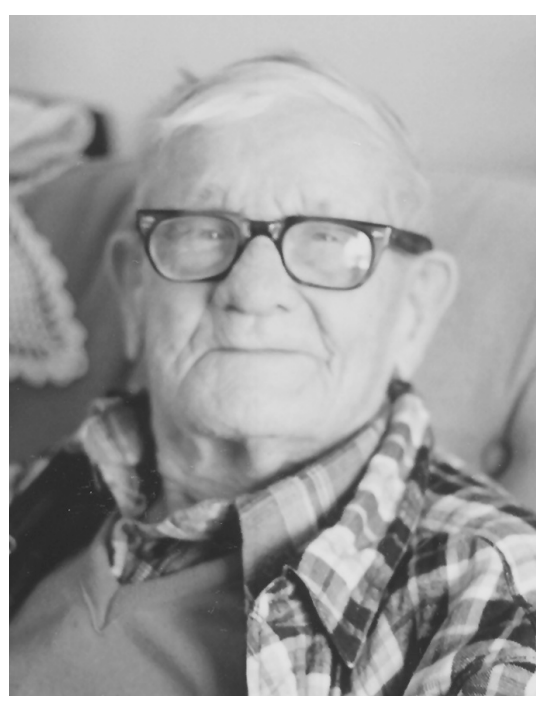

Alain Kelly

Source : photographie de Ronald Labelle, 2002 sances sur les coutumes acadiennes du passé, les légendes et croyances traditionnelles, l'histoire locale, les anciennes techniques de pêche et d'agriculture, ainsi que sur la vie dans les chantiers forestiers. C'est seulement à l'âge de 100 ans que sa mémoire a commencé à faire défaillance et il a quand même réussi à chanter au Miramichi Folksong Festival chaque année jusqu'à l'âge de 104 ans.

Alain Kelly est décédé une semaine après avoir atteint l'âge vénérable de 105 ans. Il était un des derniers témoins du mouvement de colonisation sur les terres de la couronne au Nouveau-Brunswick datant du début des années 1930. Sa vie a fait l'objet de ma thèse de doctorat intitulée «"J'avais le pouvoir d'en haut" : la représentation de l'identité dans le témoignage autobiographique d'Alain Kelly» (Université Laval, 2001). Mais on se rappellera surtout de lui en tant que chanteur traditionnel. Ayant participé au Miramichi Folksong Festival dès sa fondation en 1958, il a connu de nombreux 
folkloristes, tant des francophones comme le père Anselme Chiasson et Francine Brunel-Reeves que des anglophones comme Helen Creighton de la Nouvelle-Écosse et Edward Ives, le fondateur du Maine Folklife Center à Orono. Des interprètes de la chanson, dont Alan Mills, lui avaient aussi rendu visite, afin d'alimenter leur répertoire traditionnel.

Kelly avait été introduit très jeune à la chanson folklorique dans son village natal de Pointe-Sapin ; il entendit d'abord les chansons irlandaises de son père William et ensuite celles chantées en français dans les veillées villageoises. Mais la plupart de son répertoire a été acquis pendant les années 1920 et 1930, alors qu'il travaillait dans les chantiers forestiers de la Miramichi, côtoyant des bûcherons acadiens et anglophones du NouveauBrunswick, ainsi que de nombreux autres venant du Québec, de l'Île-duPrince-Édouard et même de Terre-Neuve. Il apprenait tout, n'ayant qu'à entendre une chanson une ou deux fois pour la retenir. Dès son premier hiver dans les chantiers, Kelly avait été plongé dans un milieu où la chanson occupait une place de choix. Le samedi soir et le dimanche, les bûcherons écoutaient leurs compagnons de travail chanter tantôt des complaintes tragiques, tantôt des pièces comiques. Il y avait souvent cinq ou six bons chanteurs dans un camp et chacun s'exécutait à son tour. En général, les francophones étaient plus nombreux que les anglophones et ils formaient aussi la majorité des chanteurs.

Parmi les chansons de tout genre qu'il connaissait, ce sont les complaintes qu'il valorisait le plus, parce qu'il sentait instinctivement qu'elles perpétuaient le souvenir de tragédies du passé. Il se donnait même comme mission de préserver ces anciennes chansons, étant encouragé en ce sens par les folkloristes qui ne cessaient de s'étonner de la richesse de son répertoire. Il chantait, par exemple, une version particulièrement longue de « La Complainte de Xavier Gallant », qui raconte le premier meurtre commis chez les Acadiens de l'Île-du-Prince-Édouard, survenu en 1812. Sa version de la complainte intitulée «La Blanche Biche» est d'une grande beauté. C'est d'ailleurs après l'avoir entendue que Francine Brunel-Reeves avait décidé de se rendre chez Alain Kelly en 1996, afin de mener une enquête filmée sur vidéo. Des folkloristes anglophones, pour leur part, sont allés cogner à se porte après l'avoir entendu chanter des ballades comme « False Knight Upon the Road» au Miramichi Folksong Festival.

Kelly donnait généreusement de son temps, mais pendant les premières années du festival, il s'est senti un peu envahi par les folkloristes et a dû trouver une façon de s'en déprendre. Edward (Sandy) Ives me raconta un jour que, lors de sa première visite chez lui vers 1960, Kelly lui expliqua qu'en tant que propriétaire d'un magasin général et père de famille, il n'avait pas le temps de chanter chaque fois qu'on venait le solliciter. Il a donc amené 
Ives dans une pièce à l'arrière du magasin où se trouvaient un magnétophone et des rubans identifiés selon les titres de chansons qu'ils contenaient. Sandy Ives l'implora d'accepter de lui chanter au moins quelques chansons, disant qu'il n'avait pas conduit mille kilomètres simplement pour s'asseoir devant un magnétophone !

Alain Kelly accordait beaucoup d'importance aux chansons religieuses, car, n'ayant eu que quelques années d'instruction formelle, sa vie spirituelle avait été nourrie par des chants comme «La Passion de Jésus-Christ: la semaine sainte », ainsi que par des chansons folkloriques à thème religieux comme « La Bergère sourde et muette ». Il avait toujours cru que cette dernière chanson devait être inspirée de faits historiques et il ne fut pas surpris lorsque je lui appris que son origine remontait à une supposée apparition de la sainte Vierge survenue le 15 août 1652 à Querrien, en Bretagne ${ }^{1}$. Même une chanson imprégnée du surnaturel comme "Le Blasphémateur transformé en chien » le laissait songeur, car sa conception du monde accordait une grande place au miracle et au surnaturel. Comme bien des Acadiens de son époque, il croyait aux signes venant de l'au-delà et il cherchait la protection divine. Il était convaincu d'avoir senti et entendu le diable pendant une nuit passée dans une maison où avait été commis un meurtre des années auparavant. Il a toujours cru aussi qu'une grave infection qui a nécessité l'amputation d'une partie de son pied gauche était le résultat d'un sort jeté par une Amérindienne suite à un tort qu'il lui aurait causé dans sa jeunesse.

Le monde dans lequel Alain Kelly avait grandi était marqué par la misère dont souffraient la plupart des habitants de la côte acadienne. Vivant dans la pauvreté extrême, la faim et la maladie les guettaient toujours. C'était certainement le cas chez les Kelly, qui ne possédaient même pas de logis au début du $\mathrm{XX}^{\mathrm{e}}$ siècle, car William Kelly avait été mis à la porte par le propriétaire de la ferme où il avait demeuré et travaillé. Il est difficile de comprendre aujourd'hui le degré de misère qu'ont connu les Acadiens et autres habitants de la côte jusqu'aux années 1940. Je suis privilégié d'avoir entretenu une relation d'amitié avec Alain Kelly pendant près de trente ans et d'avoir pu recueillir son récit de vie qu'il m'a livré d'une façon extrêmement précise et détaillée. Il m'a longuement décrit comment les habitants de l'est du NouveauBrunswick vivaient dans un état de privations matérielles presque totales, étant exploités sans merci par quelques riches entrepreneurs.

Pour Alain Kelly et les autres de sa génération, la seule façon d'en sortir était d'être fort et de traverser les épreuves à l'aide d'une foi divine inébranlable. Ce monde dans lequel il a vécu ses années de formation n'existe plus, mais grâce à lui, nous pouvons connaître le folklore de cette époque et le contexte social dans lequel il existait. En tant qu'informateur par excellence,

1. Joseph Le Floc’h, « La Musique et le rite dans la chanson traditionnelle française », p. 20. 
Kelly a donc apporté une contribution précieuse à l'ethnologie acadienne et le legs qui se trouve maintenant aux archives d'ethnologie et de folklore du Centre d'études acadiennes Anselme-Chiasson continuera longtemps à nous éclairer au sujet de la mentalité et de la culture de nos ancêtres.

RonALD LABELLE Université de Moncton 